\title{
Expression of ezrin is associated with invasion and dedifferentiation of hepatitis $B$ related hepatocellular carcinoma Chun-Nan Yeh ${ }^{\dagger 1}$, See-Tong Pang ${ }^{\dagger 2}$, Tsung-Wen Chen ${ }^{1}$, Ren-Ching Wu ${ }^{3}$, Wen- Hui Weng ${ }^{4}$ and Miin-Fu Chen*1
}

\begin{abstract}
Address: ${ }^{1}$ Department of Surgery, Chang Gung Memorial Hospital; Chang Gung University, Taoyuan, Taiwan, Republic of China, ${ }^{2}$ Department of Urology, Chang Gung Memorial Hospital; Chang Gung University, Taoyuan, Taiwan, Republic of China, ${ }^{3}$ Department of Pathology, Chang Gung Memorial Hospital; Chang Gung University, Taoyuan, Taiwan, Republic of China and ${ }^{4}$ Department of Chemical Engineering and Biotechnology, National Taipei University of Technology, Taipei City, Taiwan, Republic of China
\end{abstract}

Email: Chun-Nan Yeh - ycn@adm.cgmh.org.tw; See-Tong Pang - ycn@adm.cgmh.org.tw; Tsung-Wen Chen - ycn@adm.cgmh.org.tw; RenChingWu - ycn@adm.cgmh.org.tw; Wen-Hui Weng - ycn@adm.cgmh.org.tw; Miin-Fu Chen* - ycn@adm.cgmh.org.tw

* Corresponding author †Equal contributors

Published: 15 July 2009

BMC Cancer 2009, 9:233 doi:10.1 186/147/-2407-9-233
Received: I October 2008

Accepted: 15 July 2009

This article is available from: http://www.biomedcentral.com/I47I-2407/9/233

(c) 2009 Yeh et al; licensee BioMed Central Ltd.

This is an Open Access article distributed under the terms of the Creative Commons Attribution License (http://creativecommons.org/licenses/by/2.0), which permits unrestricted use, distribution, and reproduction in any medium, provided the original work is properly cited.

\begin{abstract}
Background: Hepatocellular carcinoma (HCC) is the fifth most common malignancy in the world and constitutes the leading cause of cancer-related death among men, and second among women in Taiwan. Liver cirrhosis and HCC are relatively prevalent, and $80 \%$ to $85 \%$ of the patients with these conditions have positive results for hepatitis B surface antigen in Taiwan. Only $5 \%$ of the general population is seronegative for all hepatititis B virus (HBV) markers. This is the first study to determine the role of ezrin upon HBV HCC cell and patients with HBV HCC undergoing hepatectomy
\end{abstract}

Methods: Immunohistochemical study with ezrin in 104 human HBV-HCC cases were carried out to investigate its association with the clinicopathological features and the outcomes of $104 \mathrm{HBV}$ HCC patients undergoing hepatetomy. In addition, DNA constructs including the wild type ezrin (wt-ezrin) and mutant ezrin Tyr353 (Y353) were transfected into Hep3B cell to study its role in tumor invasion and differentiation.

Results: HBV HCC patients with ezrin over-expression independently have smaller tumor size, cirrhotic liver background, poor tumor differentiation, and more vascular invasion. Ezrin expression status has no impact on survival for HBV-HCC patients undergoing hepatectomy. The in vitro assay showed that wt-ezrin Hep3B cells have a significant higher level of AFP secretion and higher invasion ability as compared with the control and Y353- ezrin Hep3B cells.

Conclusion: Ezrin over-expression contributed to de-differentiation and invasion of HBV-HCC cell. HBV-HCC patients with ezrin over-expression were independently associated with tumor with smaller size, cirrhotic liver background, poor differentiation, and vascular invasion. 


\section{Background}

Hepatocellular carcinoma (HCC) is a common disease in Taiwan, with an age-adjusted incidence of 28.7 per 100000 of the population per annum. It continues to be the leading cause of cancer-related death among men and the second among women [1]. In Taiwan, liver cirrhosis and HCC are relatively prevalent, and $80 \%$ to $85 \%$ of the patients with these conditions have positive results for hepatitis B surface antigen (HBsAg) [2-4]. Only 5\% to 6\% of the general population is seronegative for all $\mathrm{HBV}$ markers [2-4]. Our previous study revealed the positive rates of HBsAg and hepatitis $\mathrm{C}$ virus antibody were $61.9 \%$ and $35.3 \%$, respectively, in patients with HCC undergoing hepatic resection [5].

The recent identification of ezrin, the product of the Villin 2 gene, as a crucial molecule in the dissemination of two pediatric tumors adds new protein to the list of metastasis-associated molecules [6,7]. Ezrin is a member of the ERM (ezrin-radixin-moesin) cytoskeleton-associated protein family [8], sharing a homology with the amino-terminal membrane-binding domain of erythrocyte band 4.1 and possessing membrane-cytoskeleton linking functions [9]. Ezrin plays a positive role in maintaining cell shape and polarity and participates in cell migration, signaling, growth regulation, and differentiation [10]. Ezrin can interact with several membrane proteins, including CD44 [11], CD43 [11], intercellular adhesion molecule-1 and intercellular adhesion molecule-2, and phosphatidylinositol [4,5]-bisphosphate [12]. In addition, ezrin can signal cell survival through the phosphatidylinositol 3kinase/Akt pathway [13]. Ezrin is also actively involved in regulating the growth and metastatic capacity of cancer. Over-expression of ezrin has been detected in several human epithelial tumors, including prostate cancer $[14,15]$, brain hemangioblastoma [16], uterine endometrioid adenocarcinoma [17], osteosarcoma [18], and uveal malignant melanoma [19].

However, the role of ezrin on HBV-HCC is unknown. The aim of this study was to help to determine the role of ezrin upon patients with HBV HCC undergoing hepatectomy in terms of demographics, laboratory data, operative findings, pathological features, and survival. In addition, in vitro study using DNA constructs including the wild type ezrin (wt-ezrin) and Tyr353 mutant ezrin (Y353-ezrin) were transfected into Hep3B cell to study its role in tumor invasion and differentiation.

\section{Methods}

\section{Clinicopathological features of HBV HCC patients}

From 1985 to 2001, 104 HBV HCC patients undergoing hepatectomy at department of surgery, Chang Gung Memorial Hospital, Taipei, Taiwan were investigated. Laboratory tests were performed on the day before surgery.
Child grading was used to classify the severity of cirrhosis of HBV HCC patients. The study was approved by the local institutional review board (IRB) of Chang Gung Memorial Hospital in the genetic transfection and clinical study and all patients gave informed consent before taking part in the study for immunostaining (No: 94-955B). Histopathological findings of HCC were divided into four grades according to Edmondson and Stainer's system [20]. Grades I and II were conditioned as low-grade HCC, and grades III and IV as high-grade.

\section{Follow-up study}

Follow-up evaluation involved clinical physical examinations and blood chemistry tests at each visit. The remnant liver was examined using abdominal ultrasonography (US) every three months, and the serum $\alpha$-fetoprotein (AFP) was measured by radioimmunoassay at least every 3 months. When US detected a new lesion, or elevated AFP was noted, an abdominal CT, MRI, or liver scan was performed for confirmation. Chest $\mathrm{x}$-ray examination was performed every 6 months for pulmonary metastasis evaluation. If a patient complained of bone pain, a bone scan was performed for the detection of metastasis. If any of the above procedures indicated recurrence, the patient was readmitted for further investigation, including an angiographic evaluation or MRI.

\section{Cell culture}

Hep 3B, a stable hepatitis-B related human HCC cell line containing an integrated hepatitis $B$ virus genome, was routinely maintained in DMEM medium. This medium contains $5 \mathrm{mg} / \mathrm{mL}$ phenol red and supplemented with $10 \%$ fetal bovine serum (FBS), 2 mM L-glutamine and 50 $\mathrm{mg} / \mathrm{mL}$ each of penicillin and streptomycin (all purchased from Life Technologies Ltd, Paisley, UK).

\section{Mutagenesis and cell transfection}

An expression construct with VSV-G-tagged human wild type ezrin (wt-ezrin) and Y353 mutant ezrin were a kindly provided by Professor Monique Arpin [13]. Hep 3B cell was seeded at a density of $1.5 \times 10^{6} / 60 \mathrm{~mm}$ dish in $2 \mathrm{ml}$ of DMEM medium one day before transfection. Transfection of ezrin expression constructs was performed using Lipofectamine 2000 reagent (Invitrogen, Carlsbad, CA) according to the manufacturer instructions. Neomycin was added into the culture medium for selecting positively transfected clones.

\section{Western Blotting}

Hep $3 \mathrm{~B}$ cell was cultivated and treated as described above. The cells were harvested using RIPA buffer with protease inhibitor. Protein concentration was determined with BCA Protein Assay Reagent (Pierce, USA). Equal amounts of protein were denatured with loading buffer, separated on a $12 \%$ SDS-PAGE gel (Invitrogen Life Technologies, 
UK) and blotted onto a PVDF membrane (Amersham, Life Science, UK). Membranes were then blocked in a Trisbuffered saline solution with 5\% BSA or nonfat dry milk and probed with primary antibody. The membranes were further probed with HRP-conjugated goat anti-mouse/ anti-rabbit to visualize band. Mouse monoclonal antihuman ezrin antibody was obtained from company (Ab1; Neomarker, Lab Vision, CA, USA) and mouse anti-VSV$\mathrm{G}$ antibody was obtained from Roche.

\section{Matrigel invasive assay}

Matrigel was purchased from Becton Dickinson (San Jose, CA, USA) and stored at $-20^{\circ} \mathrm{C}$. After thawing at $4{ }^{\circ} \mathrm{C}$ overnight, matrigel was diluted in serum free-cold DMEM medium and 50 ul were evenly inoculated onto upper chamber of $6.5 \mathrm{~mm}$ transwell membrane and allowed to form a gel at $37^{\circ} \mathrm{C}$ incubator. The Matrigel invasive assay was carried out according to the manufacturer's instructions. A $0.2 \mathrm{~mL}$ of aliquot of cell suspension containing $3.3 \times 10^{5}$ cells was seeded on the upper chamber of Matrigel coated transwell filter ( 8 um pore). The serumcontaining medium was added to the lower chamber and incubated for $48 \mathrm{hrs}$ at $37^{\circ} \mathrm{C}$ in a humidified atmosphere of $5 \% \mathrm{CO}_{2}$. Non-invading cells that remained on the upper surface of the filter were removed with cotton wool; the cells that appeared on the lower surface of the filter were fixed in $4 \%$ paraformaldehyde, stained with hematoxylin and counted under a microscope. Each assay was carried out in triplicate in three different occasions.

\section{Differentiation assay}

Detection of alpha-fetoprotein by radioimmunoassay and albumin by biochemical test: $5 \times 10^{6}$ cells in $10 \mathrm{ml}$ medium were plated in $100 \mathrm{~mm}$ culture plate. Incubate cells at $37^{\circ} \mathrm{C}$ in a $\mathrm{CO}_{2}$ incubator for 40 hours. The supernatant was harvested for test and cell number was counted for adjustment.

\section{Immunohistochemistry}

From the archives of Chang Gung Memorial Hospital (from 1985 to 2001), each case of HCC was selected based on the availability of sufficient quantities of tumor cells. H\&E-stained slides from each case were reviewed. $4 \mu \mathrm{m}$ section of 104 patients with HBV related HCCs from formalin-fixed, paraffin-embedded tissue were stained for ezrin. The primary antibody ezrin was diluted to 1:1500 and added to the slides to be incubated overnight at $4{ }^{\circ} \mathrm{C}$. The slides were then washed three times for 5 minutes in TBST before visualization with the "DAKO LSAB2 System, Peroxidase" DAKO A/S, No K0675). Control slides were incubated with secondary antibody only. After washing three times for 5 minutes each in TBST, the slides were mounted and analyzed under microscope by authors blindly.

\section{Statistical analysis}

All data are presented as percentage of patients or mean with standard deviation. Numerical data were compared by independent two-sample $\mathrm{t}$ tests. Nominal data were compared by Pearson chi-square test, Fisher exact test or multiple forward stepwise logistic regression test when appropriate. Survival was calculated and plots constructed according to the Kaplan-Meier method. Furthermore, the log-rank test was performed for a statistical univariate analysis of prognostic variables. All statistical analyses were performed using the SPSS computer software package (Version 10.0, Chicago, IL, USA). A value of $\mathrm{P} \leq 0.05$ was considered significant.

\section{Results \\ Clinicopathological features of 104 HBV HCC patients after hepatectomy regarding ezrin staining status}

Table 1 summarized the clinicopathological features of $104 \mathrm{HBV}$ HCC patients, including 86 men and 18 women with a median age of 53.4 years. All the 104 patients were positive for hepatitis B surface antigen. Among them, 67 out of $104(64.4 \%)$ patients had underlying cirrhosis and all the 67 patients had Child A grading. 64 out of 104 patients had high grade HCC (grade III and IV) (61.5\%),

Table I: Clinicopathological features of 104 patients with hepatitis-B HCC undergoing hepatectomy

\begin{tabular}{ll}
\hline Demographic data & \\
\hline Male: Female & $86: 18$ \\
Age (years) (median; range) & $53.4 ; 23-79$ \\
AFP (ng/ml) (median; range) & $143 ; 3-1000$ I \\
HBsAg positive, (\%) & $104(100)$ \\
Tumor size (cm) (median; range) & $5.0 ; 1.5-20.5$ \\
Liver cirrhosis (+) (\%) & $67 / 104(64.4 \%)$ \\
\hline Child grading & \\
\hline A & \\
B & 67 \\
\hline Extent of hepatic resection & 0 \\
\hline Extended right lobectomy & \\
Right lobectomy & \\
Extended left lobectomy & $10(9.6 \%)$ \\
Left lobectomy & $17(16.3 \%)$ \\
Partial hepatectomy & $5(4.8 \%)$ \\
\hline Pathological features & $13(12.5 \%)$ \\
& $59(56.7 \%)$ \\
\hline Tumor differentiation (I, II, III, and IV) & \\
Capsular invasion & $3,37,54,10$ \\
Vascular invasion & $66 / 104(63.5 \%)$ \\
Satellite lesions & $25 / 104(24.0 \%)$ \\
Recurrence & $27 / 104(26.0 \%)$ \\
\hline & $68 / 104(65.4 \%)$ \\
\hline L & \\
\hline
\end{tabular}

HBsAg: hepatitis B surface antigen 
66 capsular invasion $(63.5 \%), 25$ vascular invasion (24.0\%), 27 satellite lesions (26.0\%), and 68 recurrence after hepatectomy (65.3\%). Regarding staining status of ezrin, the HBV-HCC patients with positive ezrin immunoreactivity had smaller tumor size, higher frequency of cirrhosis, tumor de-differentiation, satellite lesions, and vascular invasion. Multivariate forward logistic regression analysis showed that small tumor size, cirrhotic liver, poor differentiation, and vascular invasion are the four independent factors associated with HBV HCC patients with positive ezrin immunoreactivity (Table 2 and 3 ).

\section{Positive fluorescent and western blotting of ezrin-vsvg expressed in Hep $3 B$ cells}

Figure 1 upper row illustrated positive fluorescent staining of VSVG in the wt-ezrin and Y-353 Hep 3B cells, indicating successful transfection of ezrin into Hep 3B cells. Figure 1 lower row depicted western blotting of VSVG expression in the wt-ezrin and Y353-ezrin Hep 3B cells, meaning successful functional expression of ezrin construct in the transfected Hep 3B cells.

\section{Ezrin Is Important for the Matrigel Invasion of Hep 3B Cell Lines}

We studied the possibility of ezrin being involved in the regulation of the invasive properties of Hep3B. Figure 2 illustrates that ezrin transfected Hep 3B cells have significant higher invasiveness than Hep 3B cells. However, inhibition of ezrin function as shown in theY353-ezrin clones has significantly reduced the invasion of Hep 3B.

\section{Determination of AFP and ALB concentration in the extracellular medium}

AFP and ALB in the extracellular medium is produced and released into extracellular fluids by cells. The appearance of AFP and ALB in the medium further confirms tumor cell de-differentiation and differentiation, respectively. In our experiments, wt-ezrin Hep 3B cell secreted significant higher AFP level when compared with Hep 3B cell, indicating ezrin is associated with de-differentiation of Hep 3B cells. But Y353-ezrin clones secreted significant lower AFP level when compared with wt-ezrin clones and the control Hep 3B cells (Figure 3). Albumin level is too low to be detected.

\section{Immunohistochemistry}

Figure 4A illustrated that ezrin is not stained in the normal hepatocyte but positive in the lymphocyte and cholangiocyte (cholangiocyte and lymphocyte can be used as internal positive control). Among the $104 \mathrm{HBV}$ HCC patients undergoing hepatectomy, 27 (29.6\%) had variable positive ezrin immunoreactivity in the cytoplasm of the tumor cell (Figure 4B, C, and 4D).

\section{Survival analysis of HBV-HCC patients undergoing hepatectomy in terms of ezrin staining status}

All of the 104 HBV-HCC patients undergoing hepatectomy were closely followed at regular intervals until death or until the time of this writing. The median follow-up period of the $104 \mathrm{HBV}-\mathrm{HCC}$ patients undergoing hepatectomy is 36.4 months (range: $1.05-134.0$ months). Similar disease-free survival (DFS) and overall survival (OS) rate were observed between the ezrin positive and ezrin negative HBV-HCC patients after hepatectomy. The 1-, 3, and 5-year DFS rates between ezrin positive and ezrin negative $\mathrm{HBV}-\mathrm{HCC}$ patients after hepatectomy are similar. Furthermore, the 1-, 3-, and 5-year OS rate between the ezrin positive and ezrin negative HBV-HCC patients after hepatectomy are also similar.

\section{Discussion and Conclusion}

Ezrin is a key signaling molecule that regulates cell survival, adhesion, migration, and invasion, which plays a role of linker protein with capacity to bind membrane proteins and the actin cytoskeleton, such interaction provides an intracellular scaffold for the formation of special-

Table 2: Clinicopathological features between Ezrin (+) and Ezrin (-) hepatitis-B HCC patients

\begin{tabular}{|c|c|c|c|}
\hline & $E(+)(n=27)(\%)$ & $E(-)(n=77)(\%)$ & $P$ \\
\hline Age (years) & $51.1 \pm 13.3$ & $52.6 \pm 13.4$ & 0.631 \\
\hline Gender (M:F) & $24: 3$ & $62: 15$ & 0.323 \\
\hline Tumor size $(\mathrm{cm})$ & $5.0 \pm 4.3$ & $7.0 \pm 4.7$ & 0.049 \\
\hline $\operatorname{AFP}(\mathrm{ng} / \mathrm{ml})$ & $1288.1 \pm 2898.0$ & $2215.3 \pm 3701.7$ & 0.201 \\
\hline Cirrhosis (+) & $23(85.2)$ & $44(58.7)$ & 0.013 \\
\hline Grading (Edmonson and Stainer) & & & 0.044 \\
\hline Low-grade $(I+I I)$ & $6(22.2)$ & $34(44.2)$ & \\
\hline High-grade (III+ IV) & $21(77.8)$ & $43(55.8)$ & \\
\hline Capsule formation $(+)$ & $16(58.9)$ & $50(64.9)$ & 0.642 \\
\hline Vascular invasion $(+)$ & $12(44.4)$ & $13(16.9)$ & 0.004 \\
\hline Satellite lesions $(+)$ & II (40.7) & $16(21.3)$ & 0.050 \\
\hline Recurrence $(+)$ & $17(63.0)$ & $51(66.2)$ & 0.759 \\
\hline
\end{tabular}

M: male; F: female; AFP: $\alpha$-fetoprotein 
Table 3: Multiple forward stepwise logistic regression analysis of clinicopathological features in 104 surgical resected hepatitis-B HCC cases (Ezrin (+) and Ezrin (-) group)

\begin{tabular}{lccc}
\hline & Odds ratio & $95 \% \mathrm{Cl}$ for Odds Ratio: Lower-Upper & $P$ \\
\hline Size<3 cm/ $>3 \mathrm{~cm}$ & 3.174 & $1.083-9.301$ & 0.035 \\
Cirrhosis/Non-cirrhosis & 4.464 & $1.269-15.625$ & 0.020 \\
Edmonson-Steiner & & & 0.027 \\
Gr III+IV/Gr I+II & 3.759 & $1.161-12.195$ & 0.015 \\
Vascular invasion $(+) /(-)$ & 4.149 & $1.314-12.987$ & $\mathrm{NS}$ \\
Satellite lesions $(+) /(-)$ & & & \\
\hline
\end{tabular}

ized membrane domains that facilitate signal transduction through a number of growth factor receptor and adhesion molecules [21]. The main function of ezrin was first known to interact with p85, the regulatory subunit of PI3-kinase (PI3K), which was involved in determining survival of epithelial cells by activating the PI3K/Akt pathway [13]. Subsequently, the regulation of adhesion, migration and invasion were observed [21] and proved important to tumor development and progression [21]. Although, over-expression of ezrin has been detected in several human epithelial tumors, including brain hemangioblastoma [16], uterine endometrioid adenocarcinoma [17], osteosarcoma [18], and uveal malignant melanoma $[19]$, this is the first study to investigate the role of ezrin on HBV-HCC from cell level to patients with HBV-HCC undergoing hepatectomy.

HBV-HCC patients with positive ezrin immunoreactivity had smaller tumor size, higher frequency of cirrhosis, tumor dedifferentiation, satellite lesions, and vascular
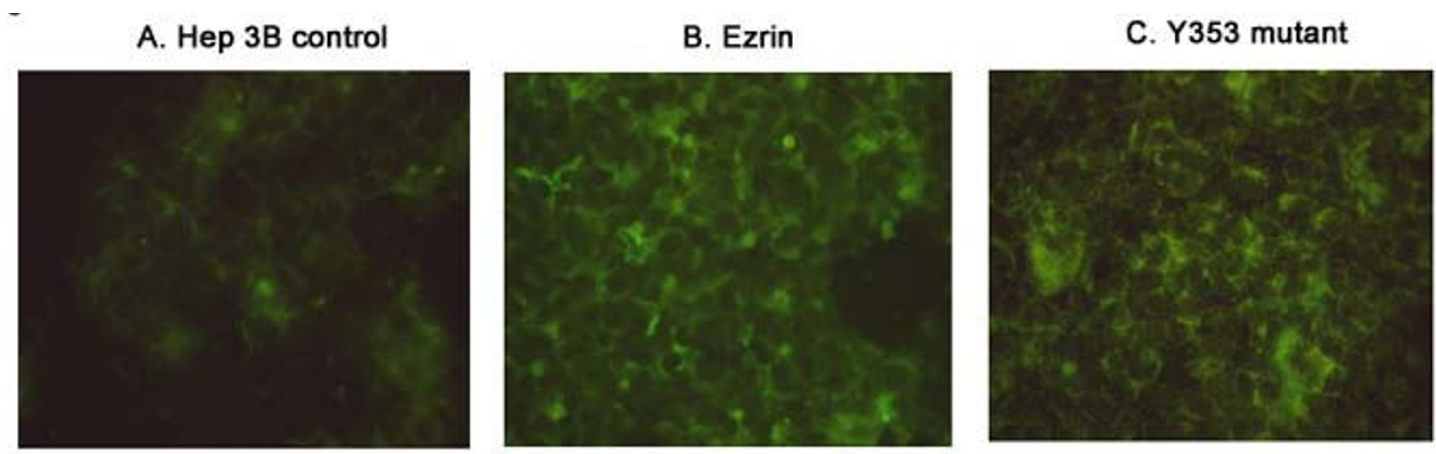

\section{Western blot analysis of ezrin-vsvg expressed in Hep 3B cells}

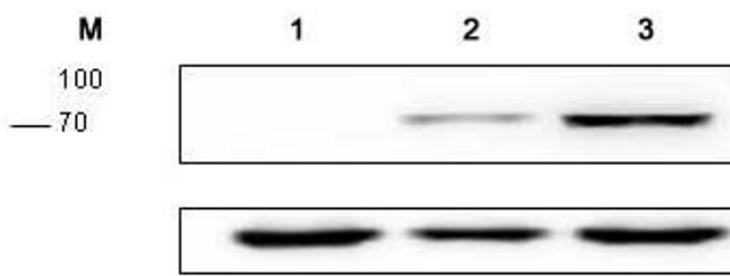

vsvg

GAPDH

Lane 1: Hep 3 B

Lane 2: Hep 3B-Ezrin-vsvg

Lane 3: Hep 3B-y353-vsvg

Loading total protein:50ug

Exposed 15 mins

\section{Figure I}

Upper row: Positive fluorescent staining of VSVG in the wild type (wt) ezrin and Y-353 Hep 3B cells, indicating successful transfection of ezrin into Hep 3B cells. Lower row: Western blotting of VSVG in the wt-ezrin and Y-353 Hep $3 B$ cells, meaning successful functional expression of ezrin construct in the transfected Hep 3B cells. 
A. Hep 3B control

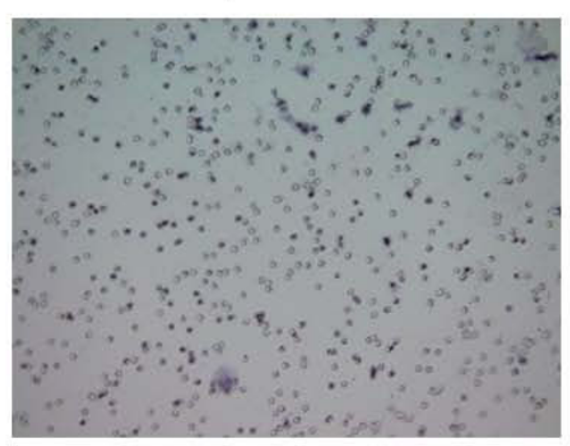

B. Ezrin

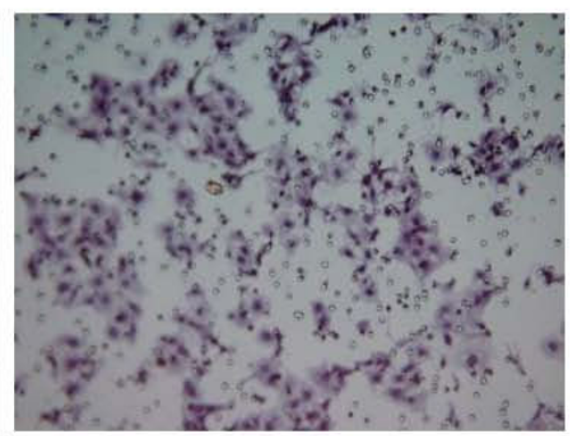

\section{Y353 mutant}

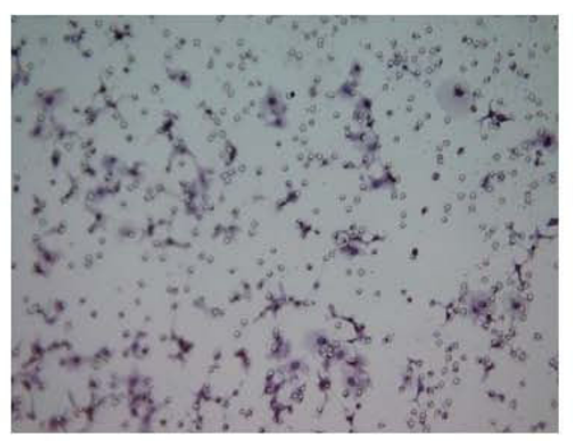

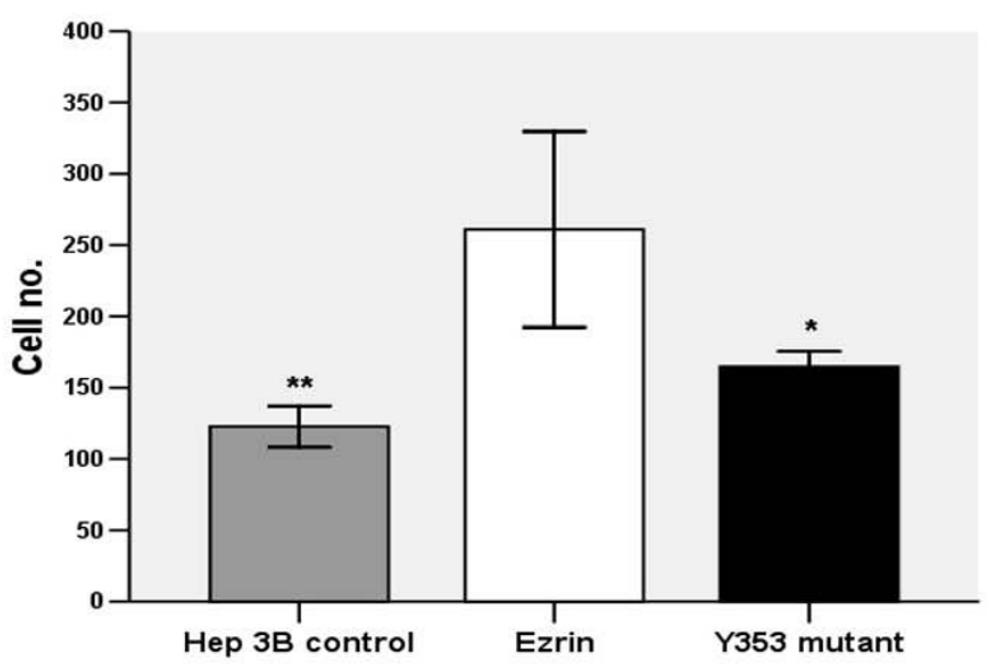

Figure 2

Wt-ezrin Hep 3B cells had significant higher invasiveness than Hep 3B cells. However, inhibition of ezrin function using Y353-ezrin mutatnt significantly reduces invasion Hep 3B cells.

invasion. Multivariate forward logistic regression analysis showed that small tumor size, cirrhotic liver, poor differentiation, and vascular invasion are the four independent factors associated with HBV HCC patients with positive ezrin immunoreactivity.

Regarding differentiation, HBV-HCC patients with positive ezrin immunoreactivity had more poor differentiation. In vitro study, we demonstrated increased activity of ezrin resulted in de-differentiation of Hep3B cell as shown increased secretion of AFP and absence of albumin secretion. Recently, the activated PI3K-Akt-mTOR pathway has recently emerged as a novel contributor to HCC development [22]. Furthermore, PI3K-Akt-mTOR pathway is associated with ezrin. Sustained activation of mTOR impaired the hepatocytic differentiation capability of these cells as shown by impaired formation of bile canaliculi, absence of polarity, and reduced secretion of A1-antitrypsin.
Decrease in the activity of mTOR observed on hepatocytic differentiation of the HepaRG cell (a cell line established from the nontumoral region of a resected HCV-associated HCC). Increased Akt-mTOR activity contributed to dedifferentiation of HepaRG cells [23]. Similar to HepaRG cells, increased activity of ezrin may increase the activity of PI3K-Akt-mTOR pathway resulting in de-differentiation of Hep3B cell. Therefore, the role of ezrin in HCC differentiation could probably mediate through the pathway of PI3K-Akt-mTOR.

Regarding invasion, increased activity of ezrin contributed to increased invasiveness of Hep3B cell as shown increased invasion of matrigel invasion assay.

On the other hand, decreased activity of ezrin by transfection of dominant-negative mutant (Y353) dramatically decreased the invasion ability of HCC cell line, because 


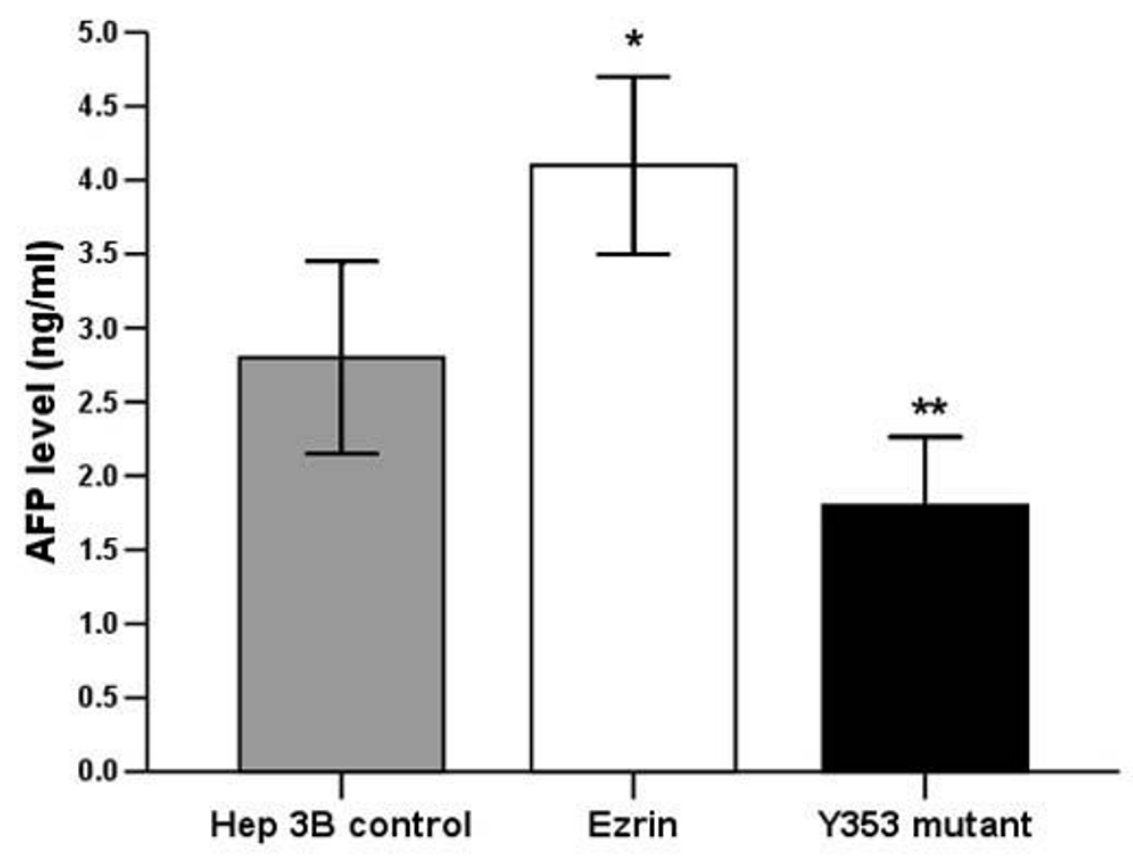

Figure 3

Wt-ezrin Hep 3B cell secreted significant higher AFP level when compared with Hep 3B cell, indicating ezrin is associated with de-differentiation of Hep 3B cells. But Y-353 Hep 3B cell secreted significant lower AFP level when compared with wt-ezrin Hep 3B and Hep 3B cells.

Y353 ezrin change the antiapoptotic signal of ezrin to apoptosis [13]. In experimental invasion assay, demonstrating that ezrin over-expression is sufficient to confer metastatic capability. HBV-HCC patients with positive ezrin immunoreactivity had more vascular invasion but no higher rate of recurrence. This contradiction could be explained by HBV-HCC patients with positive ezrin immunoreactivity having smaller tumor size.

Regarding survival, ezrin expression was not an independent prognostic factor for HBV-HCC patients after hepatectomy with long-term disease-free survival and overall survival. Small tumor size, cirrhotic liver, poor differentiation, and vascular invasion are the four independent factors associated with HBV HCC patients with positive ezrin immunoreactivity.

Our previous study has demonstrated that overall survival and disease-free survival for small sized HCC is better than large HCC [24]. Patients with long-term disease-free survival after hepatectomy usually had lower rate of underlying liver cirrhosis. The effect of underlying cirrhosis in the non-tumorous liver on risk of recurrence after HCC resection is, however, controversial, although cirrhosis was reported to be a significant risk factor for recur- rence in the remaining liver, owing to a predisposition to multicentric hepatocarcinogenesis [25]. In addition, when the patients with low-grade HCC after hepatectomy was related with the long-term disease-free survival, however, the prognostic significance of histological grading of HCC on risk of recurrence is debatable [26]. Vascular invasion is the most consistently reported risk factor for recurrence after HCC resection [27]. Intrahepatic portal vein involvement is widely accepted as the mechanism for intrahepatic recurrence for HCC. Although our study shows that expression of ezrin is related to tumor differentiation status and vascular invasion ability, its expression has no significant impact on survival for our patient group. In addition, the expression of ezrin has an inversely relationship with the tumor size. This seems to be a conflicting outcome of our analysis, however, as we were looking at a group of patient who received surgical treatment, the result can not truly reflect the impact of this marker on HCC patients. In general, one would expect that, patient with smaller tumor size would have a better outcome if completely resection of the tumor can be made. Taken together, our result of ezrin expression status has no impact on survival for HBV-HCC patients who had undergone hepatectomy has to be carefully interpreted. Therefore, it would be of importance to study the expression of 


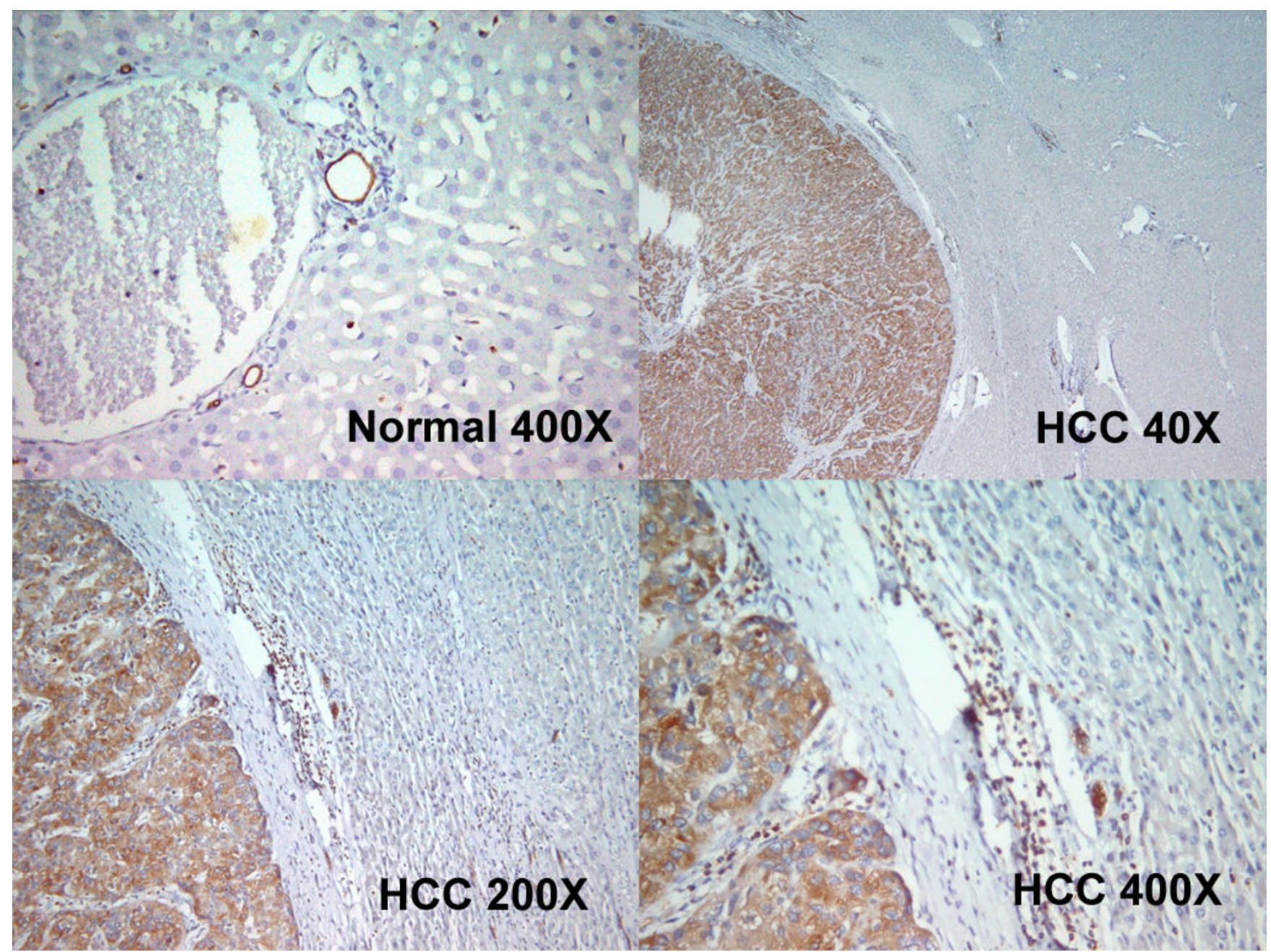

\section{Figure 4}

(A). Ezrin is not stained in the normal hepatocyte but positive in the lymphocyte and cholagiocyte (cholangiocyte and lymphocyte can be used as internal positive control). (B, C, D). Positive ezrin immunoreactivity was observed in the membrane and/or cytoplasm of the tumor cells from low power to high power field $(B, \times 40 ; C \times 200 ; D \times 400)$.

ezrin including other hepatitis related HCC patients in order to establish its role as an independent prognostic marker for HCC.

In conclusion, over-expression of ezrin in HBV-HCC patients was independently associated with small tumor size, cirrhotic liver, poor differentiation, and vascular invasion. Ezrin over-expression contributes to de-differentiation and invasion of HBV-HCC cell.

\section{Competing interests}

The authors declare that they have no competing interests.

\section{Authors' contributions}

CNY designed the experiment and drafted the manuscript. STP drafted and revised the manuscript and contributed equally to the paper as the first author CNY. TWC carried out the transfection, western blotting, and immuohistochemical staining. RCW examined the immunostaing of the slide and carried out the scoring of the immuostaing results.WHW contributed to the revision and wording of the manuscript. MFC assisted and supervised the design of the experiment and draft of the manuscript. All authors read and approved the final version of the manuscript.

\section{Acknowledgements}

I (CNY) acknowledge that this work was supported by Chang Gung Medical Research Program (CMRP) grants 34030I, 340302 and National Medical Research Program (NMRP) grants 34055I and 55005I.

\section{References}

I. Department of Health, Executive Yuan, Republic of China: Annual report of cancer registration 2006. 
2. Beasley RP, Hwang LY, Lin CC, et al.: Hepatocellular and hepatitis B virus: a prospective study of $\mathbf{2 2 7 0 7}$ men in Taiwan. Lancet I98I, 2: I I29-II 33.

3. Liaw YF, Lin DY, Chen TL, et al:: Natural course after the development of cirrhosis in patients with chronic type B hepatitis: a prospective study. Liver 1989, 9:235-24I.

4. Chen DS: From hepatitis to hepatoma: lessons from type B viral hepatitis. Science 1993, 232:369-370.

5. Chen MF, Jeng LB, Lee WC: Surgical results in patients with hepatitis virus-related hepatocellular carcinoma in Taiwan. World J Surg 2002, 26(6):742-747.

6. Yu Y, Khan J, Khanna C, Helman L, Meltzer PS, Merlino G: Expression profiling identifies the cytoskeletal organizer ezrin and the developmental homeoprotein Six-I as key metastatic regulators. Nat Med 2004, 10:175-|81.

7. Khanna C, Wan X, Bose S, Cassaday R, Olomu O, Mendoza A, Yeung C, Gorlick R, Hewitt SM, Helman L): The membrane - cytoskeleton linker ezrin is necessary for osteosarcoma metastasis. Nat Med 2004, 10:182-186.

8. Bretscher A, Edwards K, Fehon RG: ERM proteins and merlin: integrators at the cell cortex. Nat Rev Mol Cell Biol 2002, 3:586-599.

9. Takeuchi K, Kawashima A, Nagafuchi A, Tsukita S: Structural diversity of band 4.I superfamily members. J Cell Sci 1994, 107:1921-1928.

10. Brown KL, Birkenhead D, Lai JC, Li L, Li R, Johnson P: Regulation of hyaluronan binding by $F$-actin and colocalization of CD44 and phosphorylated ezrin/radixin/moesin (ERM) proteins in myeloid cells. Exp Cell Res 2005, I5; 303(2):400-I4.

II. Yonemura S, Hirao M, Doi Y, et al.: Ezrin/radixin/moesin (ERM) proteins bind to a positively charged amino acid cluster in the juxta-membrane cytoplasmic domain of CD44, CD43, and ICAM-2. J Cell Biol I998, 23; I 40(4):885-95.

12. Heiska L, Alfthan K, Gronholm M, Vilja P, Vaheri A, Carpen O: Association of ezrin with intercellular adhesion molecule-I and 2 (ICAM-I and ICAM-2). Regulation by phosphatidylinositol 4, 5-bisphosphate. J Biol Chem I998, 2I; 273(34):21893-900.

13. Gautreau A, Poullet P, Louvard D, Arpin M: Ezrin, a plasma membrane-microfilament linker, signals cell survival through the phosphatidylinositol 3-kinase/Akt pathway. Proc Natl Acad Sci USA 1999, 22; 96(13):7300-5.

14. Pang ST, Fang X, Valdman A, Norstedt G, Pousette A, Egevad L, Ekman P: Expression of ezrin in prostatic intraepithelial neoplasia. Urology 2004, 63(3):609-12.

15. Valdman A, Fang X, Pang ST, Nilsson B, Ekman P, Egevad L: Ezrin expression in prostate cancer and benign prostatic tissue. Eur Urol 2005, 48(5):852-7.

16. Bohling $\mathrm{T}$, Turunen $\mathrm{O}$, Jaaskelainen J, et al: Ezrin expression in stromal cells of capillary hemangioblastoma. An immunohistochemical survey of brain tumors. Am J Pathol 1996 I 48(2):367-73.

17. Ohtani K, Sakamoto H, Rutherford T, et al.: Ezrin, a membranecytoskeletal linking protein, is highly expressed in atypical endometrial hyperplasia and uterine endometrioid adenocarcinoma. Cancer Lett 2002, I 79(I):79-86.

18. Wang LL: Biology of osteogenic sarcoma. Cancer J 2005 , I I(4):294-305.

19. Makitie T, Carpen O, Vaheri A, Kivela T: Ezrin as a prognostic indicator and its relationship to tumor characteristics in uveal malignant melanoma. Invest Ophthalmol Vis Sci 200I, 42(II):2442-9.

20. Edmondson HA, Steiner PE: Primary carcinoma of the liver: a study of 100 cases among 48900 necropsies. Cancer 1954 , 7:462-503.

21. Hunter KW: Ezrin, a key component in tumor metastasis. Trends Mol Med 2004, 10(5):201-4.

22. Adjei $A A$, Hidalgo $M$ : Intracellular signal transduction pathway proteins as targets for cancer therapy. J Clin Oncol 2005, 23:5386-5403.

23. Parent R, Kolippakkam D, Booth G, Beretta L: Mammalian target of rapamycin activation impairs hepatocytic differentiation and targets genes moderating lipid homeostasis and hepatocellular growth. Cancer Res 2007, 67(9):4337-4345.

24. Yeh CN, Lee WC, Chen MF: Hepatic resection and prognosis for patients with hepatocellular carcinoma larger than 10 cm: two decades of experience at Chang Gung memorial hospital. Ann Surg Oncol 2003, 10(9):1070-6.

25. Yeh CN, Chen MF, Lee WC, Jeng LB: Prognosis factors of hepatic resection for hepatocellular carcinoma with cirrhosis: univariate and multivariate analysis. J Surg Oncol 2002, 8 I: 1 95-202.

26. Yeh CN, Lee WC, Chen MF, Tsay PK: Predictors of long-term disease-free survival after resection of hepatocellular carcinoma: two decades of experience at Chang Gung Memorial Hospital. Ann Surg Oncol 2003, 10(8):916-2I.

27. Poon RTP, Fan ST, Wong J: Risk factors, prevention, and management of postoperative recurrence after resection of hepatocellular carcinoma. Ann Surg 2000, 232:10-24.

\section{Pre-publication history}

The pre-publication history for this paper can be accessed here:

http://www.biomedcentral.com/1471-2407/9/233/pre pub
Publish with Biomed Central and every scientist can read your work free of charge

"BioMed Central will be the most significant development for disseminating the results of biomedical research in our lifetime. "

Sir Paul Nurse, Cancer Research UK

Your research papers will be:

- available free of charge to the entire biomedical community

- peer reviewed and published immediately upon acceptance

- cited in PubMed and archived on PubMed Central

- yours - you keep the copyright 